$\Rightarrow$ MILESTONE 19

\section{Read my mind}

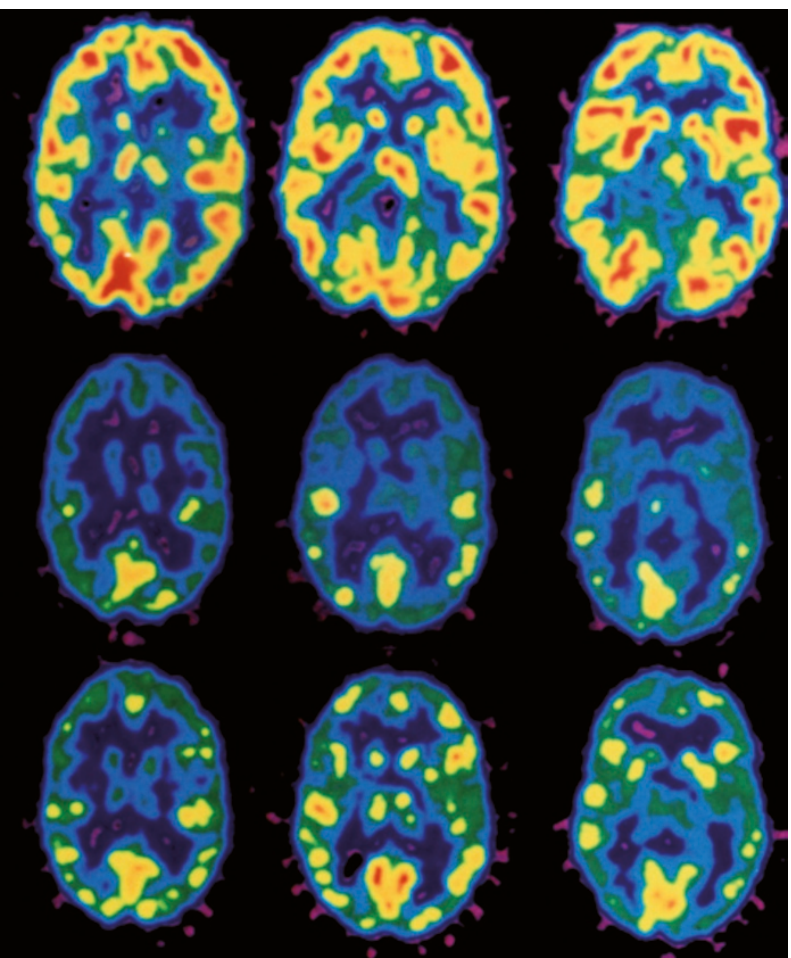

By the late 1980 s, magnetic resonance imaging (MRI; Milestone 15) had become a standard technique in hospitals and laboratories for the anatomical imaging of various tissues, from muscle to brain. However, MRI was only capable of revealing static structure and physiochemical information, but not actual function. The options for functional imaging were generally cumbersome, with low spatial resolution, and could require the injection of radioactive tracers into the bloodstream, as in positron emission tomography (PET), meaning that individual subjects could be scanned only infrequently. In 1990, however, Seiji Ogawa and colleagues published a series of breakthroughs that transformed MRI into a non-invasive and relatively inexpensive means of revealing physiological activity in the brain, sparking a revolution in the study of brain and behaviour.

Ogawa et al. exploited two

physiological phenomena that stemmed from observations made years earlier. First, in 1890, Charles Roy and Charles Sherrington had suggested that metabolic activity in the brain could be linked to vascular changes that would refresh blood supply. Later work established a more refined view: vasculature responds in an exquisitely localized fashion to bring oxygenated blood to areas of increased neural activity. This phenomenon was already being exploited in PET and other techniques. Second, Linus Pauling and Charles Coryell had reported, in 1936, that haemoglobin - the metalloprotein in red blood cells that acts as a major transporter of oxygen in humans and other species - has different magnetic properties in its oxygenated and deoxygenated forms. In three papers published in 1990, Ogawa and colleagues now showed how these two phenomena could be detected using MRI.

First, they demonstrated that changes in the level of deoxygenated haemoglobin in blood changed the proton signal from the water molecules surrounding the vessels - an effect called blood-oxygenationlevel-dependent (BOLD) contrast. Because metabolic activity in the brain involves changes in the relative levels of oxyhaemoglobin and deoxyhaemoglobin, Ogawa reasoned that it should be possible to track changes in brain activity by measuring the BOLD contrast - and in his third paper of 1990, he demonstrated exactly this. Manipulation of the brain metabolism and, hence, the blood oxygen of an anaesthetized rat - by adjusting anaesthesia or the composition of inhaled gas, or by inducing hypoglycaemia - led to changes in the BOLD contrast throughout the brain.

Two years after this ground-breaking proof of principle, three independent groups (including that of Ogawa) published, almost simultaneously, demonstrations of task-related changes in the BOLD contrast in the human brain - proving not only that this method could be translated from anaesthetized animals to awake humans, but also that it could reveal localized brain function evoked by specific stimuli, such as visual images.

BOLD-contrast imaging - or

functional MRI (fMRI) as the technique is now commonly known - quickly became a mainstay of cognitive neuroscience. It is an accessible option for measuring brain activity with relatively high spatial resolution - resolution that has improved with advances in MRI hardware and techniques, and analysis methods. From the detailed characterization of the function of human visual brain areas to the discovery of areas that are potentially involved in higher cognitive functions, such as face recognition, empathy and self-awareness, the possibilities revealed by $\mathrm{fMRI}$ seem endless.

I-han Chou, Senior Editor, Nature

ORIGINAL RESEARCH PAPERS Roy, C. S. Sherrington, C. S. On the regulation of the blood supply of the brain. J. Physiol. 11, 85-108 (1890) Pauling, L. \& Coryell, C. D. The magnetic properties and structure of hemoglobin, oxyhemoglobin and carbonmonoxyhemoglobin. Proc. Natl Acad. Sci. USA 22, 210-216 (1936)| Ogawa, S., Lee, T. M., Kay, A. R. \& Tank, D. W. Brain magnetic resonance imaging with contrast dependent on blood oxygenation. Proc. Natl Acad. Sci. USA 87, 9868-9872 (1990)|

Ogawa, S., Lee, T. M., Nayak, A. \& Glynn, P. Oxygenation-sensitive contrast in magnetic resonance image of rodent brain at high magnetic fields. Magn. Reson. Med. 14, 68-78 (1990) Ogawa, S. \& Lee, T. M. Magnetic resonance imaging of blood vessels at high fields: in vivo and in vitro measurements and image simulation. Magn. Reson. Med. 16, 9-18 (1990)| Bandettini, P. A., Wong, E. C., Hinks, R. S., Tikofsky, R. S. \& Hyde, J. S. Time course EPI of human brain function during task activation. Magn. Reson. Med. 25, 390-397 (1992) | Kwong, K. K. et al. Dynamic magnetic resonance imaging of human brain activity during primary sensory stimulation. Proc. Natl Acad. Sci. USA 89, 5675-5679 (1992)

Ogawa, S. et al. Intrinsic signal changes accompanying sensory stimulation: functional brain mapping with magnetic resonance imaging. Proc. Natl Acad. Sci. USA 89, 5951-5955 (1992) 